\title{
P-selectin increases angiotensin II-induced cardiac inflammation and fibrosis via platelet activation
}

\author{
GAIZHEN LIU $^{1}$, BIN LIANG ${ }^{1}$, XIAOSU SONG ${ }^{1}$, RUI BAI ${ }^{1}$, WEIWEI QIN ${ }^{1}$, \\ XU SUN ${ }^{2}$, YAN LU $^{3}$, YUNFEI BIAN ${ }^{1}$ and CHUANSHI XIAO ${ }^{3}$ \\ ${ }^{1}$ Department of Cardiology, The Second Hospital of Shanxi Medical University, Taiyuan, Shanxi 030001; \\ ${ }^{2}$ Department of Cardiology, Tianjin Port Hospital, Tianjin 300456; ${ }^{3}$ Department of Cardiology, \\ The First Hospital of Shanxi Medical University, Taiyuan, \\ Shanxi 030001, P.R. China
}

Received May 23, 2015; Accepted March 31, 2016

DOI: $10.3892 / \mathrm{mmr} .2016 .5186$

\begin{abstract}
Platelet activation is important in hypertension-induced cardiac inflammation and fibrosis. P-selectin expression significantly $(\mathrm{P}<0.05)$ increases when platelets are activated during hypertension. Although P-selectin recruits leukocytes to sites of inflammation, the role of P-selectin in cardiac inflammation and fibrosis remains to be elucidated. The present study aimed to investigate whether platelet-derived P-selectin promotes hypertensive cardiac inflammation and fibrosis. P-selectin knockout (P-sel KO) mice and wild-type (WT) C57BL/6 littermates were infused with angiotensin II (Ang II) at $1,500 \mathrm{ng} / \mathrm{kg} / \mathrm{min}$ for 7 days and then cross-transplanted with platelets originating from either WT or P-sel KO mice. P-selectin expression was increased in the myocardium and plasma of hypertensive mice, and the $\mathrm{P}$-sel KO mice exhibited significantly $(\mathrm{P}<0.05)$ reduced cardiac fibrosis. The fibrotic areas were markedly smaller in the hearts of P-sel KO mice compared with WT mice, as assessed by Masson's trichrome staining. In addition, $\alpha$-smooth muscle actin and transforming growth factor $\beta 1$ (TGF- $\beta 1$ ) expression levels were decreased in the P-sel KO mice, as assessed by immunohistochemistry. Following platelet transplantation into P-sel KO mice, the number of Mac-2 (galectin-3)- and TGF- $\beta 1$-positive cells was increased in mice that received WT platelets compared with those that received P-sel KO platelets, and the mRNA expression levels of collagen I and TGF- $\beta 1$
\end{abstract}

Correspondence to: Professor Yunfei Bian, Department of Cardiology, The Second Hospital of Shanxi Medical University, 382 Wuyi Road, Taiyuan, Shanxi 030001, P.R. China

E-mail: yunfeibian@sina.com

Professor Chuanshi Xiao, Department of Cardiology, The First Hospital of Shanxi Medical University, 85 Jiefang South Road, Taiyuan, Shanxi 030001, P.R. China

E-mail: 237634097@qq.com

Key words: P-selectin, platelet, hypertension, inflammation, cardiac fibrosis were also increased. The results from the present study suggest that activated platelets secrete $\mathrm{P}$-selectin to promote cardiac inflammation and fibrosis in Ang II-induced hypertension.

\section{Introduction}

Hypertension is a common clinical condition that is associated with high morbidity and mortality. The characteristics of hypertension-associated cardiac remodeling include inflammation, hypertrophy and fibrosis. Cardiac function deteriorates as the fibrosis progresses, ultimately resulting in heart failure (1). As inflammatory cells, platelets are involved in the physiological and pathological processes of numerous cardiovascular diseases. Following activation, platelets promote cardiac inflammation and fibrosis in conditions of angiotensin II (Ang II)-induced hypertension, and these effects may be prevented by a purinergic receptor P2Y12 antagonist that inhibits platelet activation (2). However, the mechanisms that underlie the role of platelet activation in initiating this process remain to be elucidated.

Platelets are generally known as central mediators in thrombosis and platelets are key in inflammation and immunity (3). Platelets interact with various types of leukocytes, including monocytes and neutrophils, to induce a systemic inflammatory response $(3,4)$. Upon activation, platelets alter their shape and gene expression pattern, expressing specific adhesion molecules (including P-selectin, cluster of differentiation 40L, glycoprotein IIb and tumor necrosis factor superfamily member 14) (4), and secreting numerous inflammatory cytokines and chemokines [including interleukin (IL)-1, platelet factor 4 and chemokine (C-C motif) ligand 5] (5). P-selectin is a key adhesion molecule, it binds to its receptor P-selectin glycoprotein ligand-1 (PSGL-1), which is expressed on the surface of blood monocytes, and mediates monocyte recruitment to sites of inflammation. The monocytes become activated, and release numerous inflammatory mediators (including monocyte chemoattractant protein-1, tumor necrosis factor- $\alpha$, IL-1 $\beta$ and IL-6), which in turn stimulate platelets to secrete more activating factors (3). This activation loop repeats and amplifies the inflammatory response. The present study hypothesized that P-selectin secreted by activated platelets may be involved 
in the inflammatory response associated with Ang II-induced hypertensive cardiac inflammation and fibrosis.

\section{Materials and methods}

Ethics statement. The animals used in the present study were bred and maintained in the Laboratory of Animal Experiments of Shanxi Medical University. The mice were fed a standard diet and were used in accordance with the US National Institutes of Health Guide for the Care and Use of Laboratory Animals (6). The present study was approved by the Institutional Animal Care and Use Committee of Shanxi Medical University.

Animal models. Male P-sel knockout (KO) mice and wild-type (WT) C57BL/6 littermates (age, 8 weeks; weight, 23-25 g; Jackson Laboratory, Sacramento, CA, USA) were used for the experiments in the present study. The mice were maintained under conditions of 50\% relative humidity, a $12 / 12 \mathrm{~h}$ light/dark cycle and $22^{\circ} \mathrm{C}$, housed separately with ad libitum access to food and water. The mice were randomized into groups (10 mice/group in P-selectin expression experiemtns, 6 mice/group in all other experiments) that were subjected to different experimental conditions. A sodium pentobarbital $(50 \mathrm{mg} / \mathrm{kg}$; Beijing Solarbio Science \& Technology Co., Ltd., Beijing, China) solution was delivered intraperitoneally to anesthetize the animals. Ang II (Sigma-Aldrich, St. Louis, MO, USA) was dissolved in a $0.01 \mathrm{~N}$ acetic acid saline solution (Tianjin Chemical Experiment Plant, Tianjin, China), and osmotic minipumps (Alzet Model 1007D; DURECT Corporation, Cupertino, CA, USA) infused with Ang II or vehicle were inserted subcutaneously into the back of the mice to deliver Ang II at a release rate of $1,500 \mathrm{ng} / \mathrm{kg} / \mathrm{min}$ for 7 days. All the treatments were well tolerated by the mice.

Blood pressure measurement. Systolic blood pressure (SBP) data were collected using a computerized mouse tail-cuff system (BP-98A; Softron Co., Ltd., Tokyo, Japan). SBP was measured prior to the initiation of the experiment and on days 4-7 of the Ang II infusion period. The mean of ten repeated values was calculated for each analysis point.

Echocardiography. The mice were anesthetized using isoflurane inhalation (Sigma-Aldrich). Cardiac function was analyzed using the Vevo 770 system (VisualSonics, Inc., Toronto, ON, Canada). The heart images were acquired in 2D mode in the parasternal short-axis view. The echocardiographic parameters were acquired in triplicate in M-mode for all the mice.

Soluble P-selectin enzyme-linked immunosorbent assay (ELISA). The concentration of plasma, which was obtained by centrifugation $\left(1760 \mathrm{x} \mathrm{g} ; 10 \mathrm{~min} ; 4^{\circ} \mathrm{C}\right)$ of soluble P-selectin was measured using a mouse P-selectin ELISA kit (ELM-Pselectin) obtained from RayBiotech, Inc. (Norcross, GA, USA) according to the manufacturer's protocols. The optical densities were read at a wavelength of $450 \mathrm{~nm}$ using an ultraviolet spectrophotometer (Varian Cary ${ }^{\circledR}$ 50; Agilent Technologies, Inc., Santa Clara, CA, USA).

Bleeding time measurement. The tail transection method was used to measure the bleeding time (7). Briefly, to record the maximum bleeding time in $900 \mathrm{sec}$, the end of bleeding was considered as the terminal point. Furthermore, the bleeding time was continuously recorded when a new arrest lasting $>30 \mathrm{sec}$ occurred and when the bleeding restarted within $30 \mathrm{sec}$.

Platelet isolation. Whole blood was collected from the hearts of anesthetized donor mice and mixed with acid citrate dextrose (ACD; Sigma-Aldrich) at a 9:1 ratio. The ACD-anticoagulated blood was centrifuged at $200 \mathrm{x}$ g for $7 \mathrm{~min}$ at room temperature to obtain platelet-rich plasma (PRP). The PRP was centrifuged at $180 \mathrm{x}$ g for a further $5 \mathrm{~min}$ at room temperature. The platelets were washed, centrifuged at $850 \mathrm{x}$ g for $12 \mathrm{~min}$ at room temperature, and resuspended in suspension buffer (minimal essential medium; Gibco; Thermo Fisher Scientific, Inc., Waltham, MA, USA) containing $10 \%$ fetal calf serum (10099-141; Gibco; Thermo Fisher Scientific, Inc.) and $300 \mathrm{ng} / \mathrm{ml}$ prostaglandin I2 (N5160; Sigma-Aldrich) at a final concentration of $1 \times 10^{8}$ platelets $/ \mathrm{ml}$.

Platelet transplantation. The 8-week-old male recipient mice were immunosuppressed using intraperitoneal busulfan, which also reduced platelet count, for 5 days $(40 \mathrm{mg} / \mathrm{kg} / \mathrm{day}$; Sigma-Aldrich). The bleeding time of the recipients was recorded as described above. The day prior to the operation, donor mouse blood samples $(8 \mathrm{ml})$ from the inner canthus were collected. A complete blood cell count of the blood samples collected in EDTA-containing tubes was performed using an automatic cell counter, and $1.5 \mathrm{ml}\left(10^{8} / \mathrm{ml}\right)$ platelets were injected into the recipient mice via the tail vein (8).

The division of the group in platelet transplantation. The mice were divided into 5 groups of 6 mice, as follows: i) WT recipient mice transplanted with WT platelets and infused with Ang II (WT-WT, positive control); ii) P-sel KO recipient mice transplanted with WT platelets and infused with Ang II (KO-WT, to assess the role of platelet-derived P-selectin); iii) WT recipient mice transplanted with $\mathrm{P}$-sel $\mathrm{KO}$ platelets and infused with Ang II (WT-KO, to assess the role of endothelium-derived P-selectin); iv) P-sel KO recipient mice transplanted with P-sel KO platelets and infused with Ang II (KO-KO, positive control); 5) WT recipient mice transplanted with WT platelets and infused with saline (WT-WT', negative control).

Tissue and histology preparation. All the mice were sacrificed on day 7 with pentobarbital, and the hearts were collected, washed with a heparin-containing saline solution (Sigma-Aldrich), fixed in 10\% formalin (Sigma-Aldrich), and embedded in paraffin (Sigma-Aldrich). The paraffin-embedded fixed tissues were then cut into $5-\mu \mathrm{m}$-thick sections, placed on polylysine-coated glass slides (HL-H05-2; Nantong Hailun Bio-Medical Apparatus Manufacturing Co., Ltd., Haimen, China) and stained with hematoxylin (Sigma-Aldrich) and Masson's trichrome reagent (Beijin Solarbio Science \& Technology Co., Ltd.). The fibrotic areas were quantitated as the ratio of the area that had stained blue to the total section area using the NIS-Elements analysis program (Nikon Corporation, Tokyo, Japan). Immunohistochemistry was performed using a standard procedure as previously described (2). The sections 
were incubated overnight at $4^{\circ} \mathrm{C}$ with antibodies against transforming growth factor $\beta 1$ (TGF- $\beta 1$; rabbit polyclonal; sc-146; 1:200; Santa Cruz Biotechnology, Inc., CA, USA), $\alpha$-smooth muscle actin ( $\alpha$-SMA; rabbit polyclonal; ab66133; 1:300; Abcam, Cambridge, MA, USA), and Mac-2 (galectin-3; rabbit polyclonal; sc-20157; 1:200; Santa Cruz Biotechnology, Inc.). The images were captured using a microscope equipped with a camera (ECLIPSE 80i/90i, Nikon Corporation).

RNA extraction and reverse transcription-quantitative polymerase chain reaction ( $q P C R$ ) analysis. Total RNA was extracted from heart tissue in the $3 \mathrm{D}$ peptide hydrogel using TRIzol according to the manufacturer's protocols (Invitrogen; Thermo Fisher Scientific, Inc.). RNA was quantified using the NanoDrop 2000 spectrophotometer (Thermo Fisher Scientific, Inc.). DNase I (18068-015; Invitrogen; Thermo Fisher Scientific, Inc.) was then applied to $2 \mu \mathrm{g}$ RNA prior to reverse-transcription with MMLV reverse transcriptase from the SuperScript ${ }^{\circledR}$ III First-Strand Synthesis System and oligo (dT) primers (Invitrogen; Thermo Fisher Scientific, Inc.) according to the manufacturer's instructions. qPCR reactions were performed on an iQ5 Real-Time PCR Detection system (Bio-Rad Laboratories, Inc., Hercules, CA, USA) using SYBR Green I (Takara Bio, Inc., Otsu, Japan) with GAPDH serving as a control. The cycling conditions were as follows: $95^{\circ} \mathrm{C}$ for $2 \mathrm{~min}$, followed by 35 cycles of $95^{\circ} \mathrm{C}$ for $30 \mathrm{sec}$ and $60^{\circ} \mathrm{C}$ for $30 \mathrm{sec}$. The following primers (9) were used to amplify the fragments: Sense, 5'-GAGCGGAGAGTACTGGATC G-3' and antisense, 5'-TACTCGAACGGGAATCCATC-3' for collagen I; sense, 5'-GCCCTGGACACCAACTATTGC-3' and antisense, 5'-GGAGCGCACGATCATGTTGG-3' for TGF- $\beta 1$; sense, 5'-GCAAACAGGAATACGACGAAGC-3' and antisense, 5'-GCTTTGGGCAGGAATGATTTG-3' for $\alpha$-SMA; and sense, 5'-CCTGGAGAAACCTGCCAAGTATGA-3' and antisense, 5'-AAGCAGGAATGAGAAGAGGCTGAG-3' for GAPDH. The experiments were repeated 3 times. The quantification cycle values (Cq values) were used to calculate the fold differences using the $2-\Delta \Delta \mathrm{Cq}$ method (10).

Western blotting analysis. Protein was extracted from the heart tissue and analyzed by western blotting as previously described (11). In brief, fresh hearts were lysed with radioimmunoprecipitation assay lysis buffer and $1 \mathrm{mM}$ phenylmethane sulfonyl fluoride (Wuhan Boster Biological Technology, Ltd. Wuhan, China). Protein samples $(60 \mu \mathrm{g})$ were separated by $10 \%$ sodium dodecyl sulfate-polyacrylimide gel electrophoresis $(100 \mathrm{mV}, 90 \mathrm{~min})$. Nonspecific proteins were blocked by incubating the membrane with $5 \%$ non-fat dried milk in Tris-buffered saline containing $0.1 \%$ Tween 20 for $1 \mathrm{~h}$ at room temperature with agitation. Proteins were transferred from the gel to nitrocellulose membranes, which were incubated overnight at $4{ }^{\circ} \mathrm{C}$ with primary antibodies against GAPDH (rabbit polyclonal; CW0101M; 1:5,000; Beijing ComWin Biotech Co., Ltd., Beijing, China), $\alpha$-SMA (1:1,000; Abcam), or TGF- $\beta 1$ (1:1,000; Santa Cruz Biotechnology, Inc.). They were then incubated at room temperature for $1 \mathrm{~h}$ with mouse anti-rabbit HRP-conjugated IgG secondary antibodies (sc-2357; 1:5,000; Santa Cruz Biotechnology, Inc.). Images were captured and quantified using a Bio-Rad ChemiDoc XRS system (Bio-Rad Laboratories, Inc.) with
A
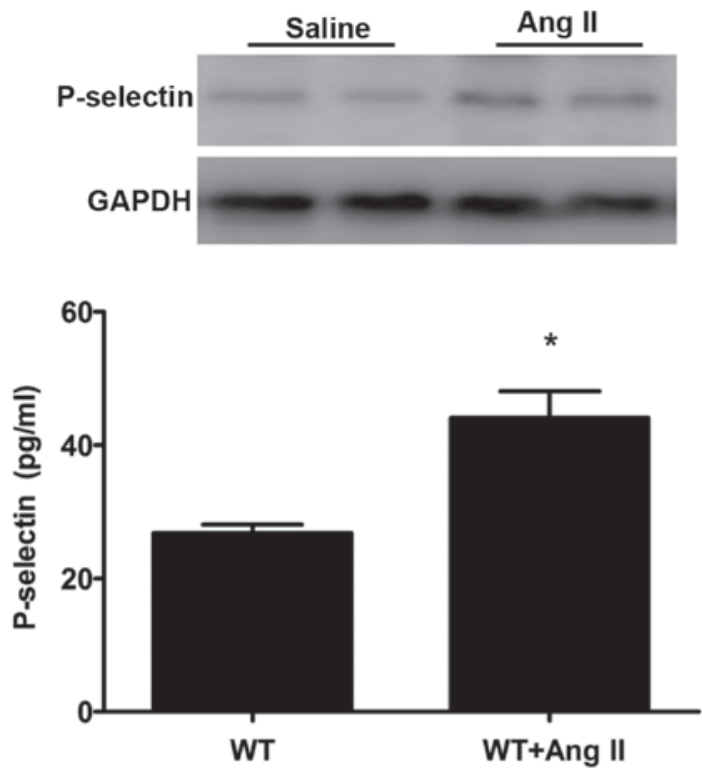

B
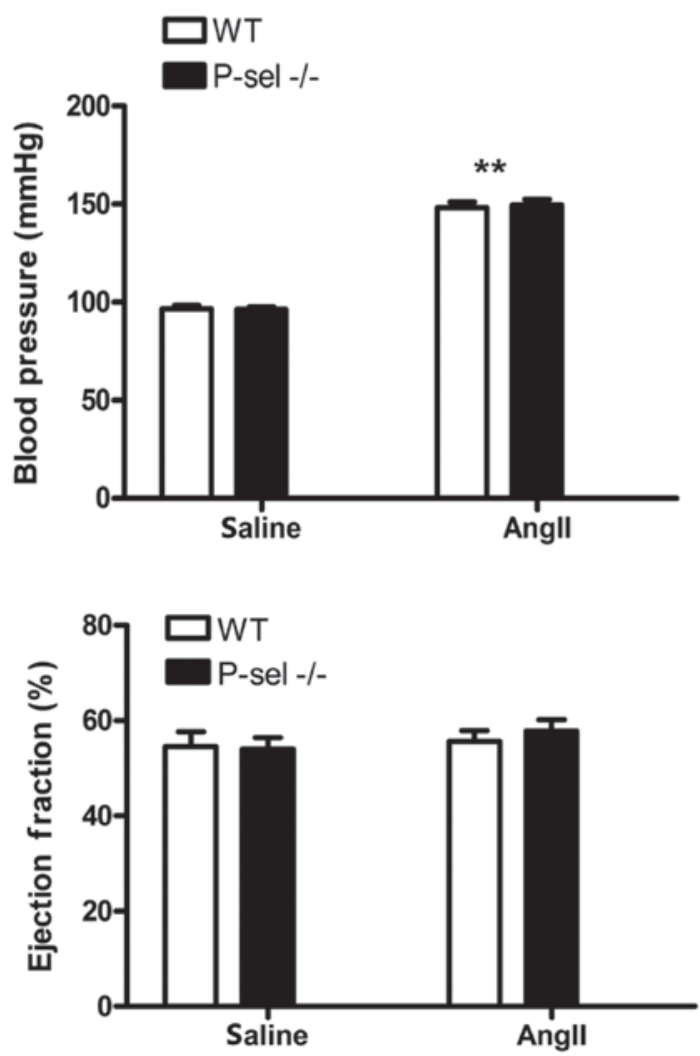

Figure 1. P-selectin expression levels were increased by Ang II in the myocardium and plasma. (A) Western blotting analysis and enzyme-linked immunosorbent assay of P-selectin expression levels in the myocardium and plasma of mice, respectively (n=10/group) following saline or Ang II infusion. (B) Systolic blood pressure and cardiac ejection fraction following Ang II infusion. The data are presented as the mean \pm standard error of the mean. ${ }^{*} \mathrm{P}<0.01$; ${ }^{* *} \mathrm{P}<0.001$ vs. saline. Ang II, angiotensin II; WT, wild-type.

Image Lab software, version 2.2 (Bio-Rad Laboratories, Inc.), and protein expression levels were normalized to GAPDH expression.

Statistical analyses. The unpaired Student's t-test was utilized to compare two groups and analysis of variance 
A
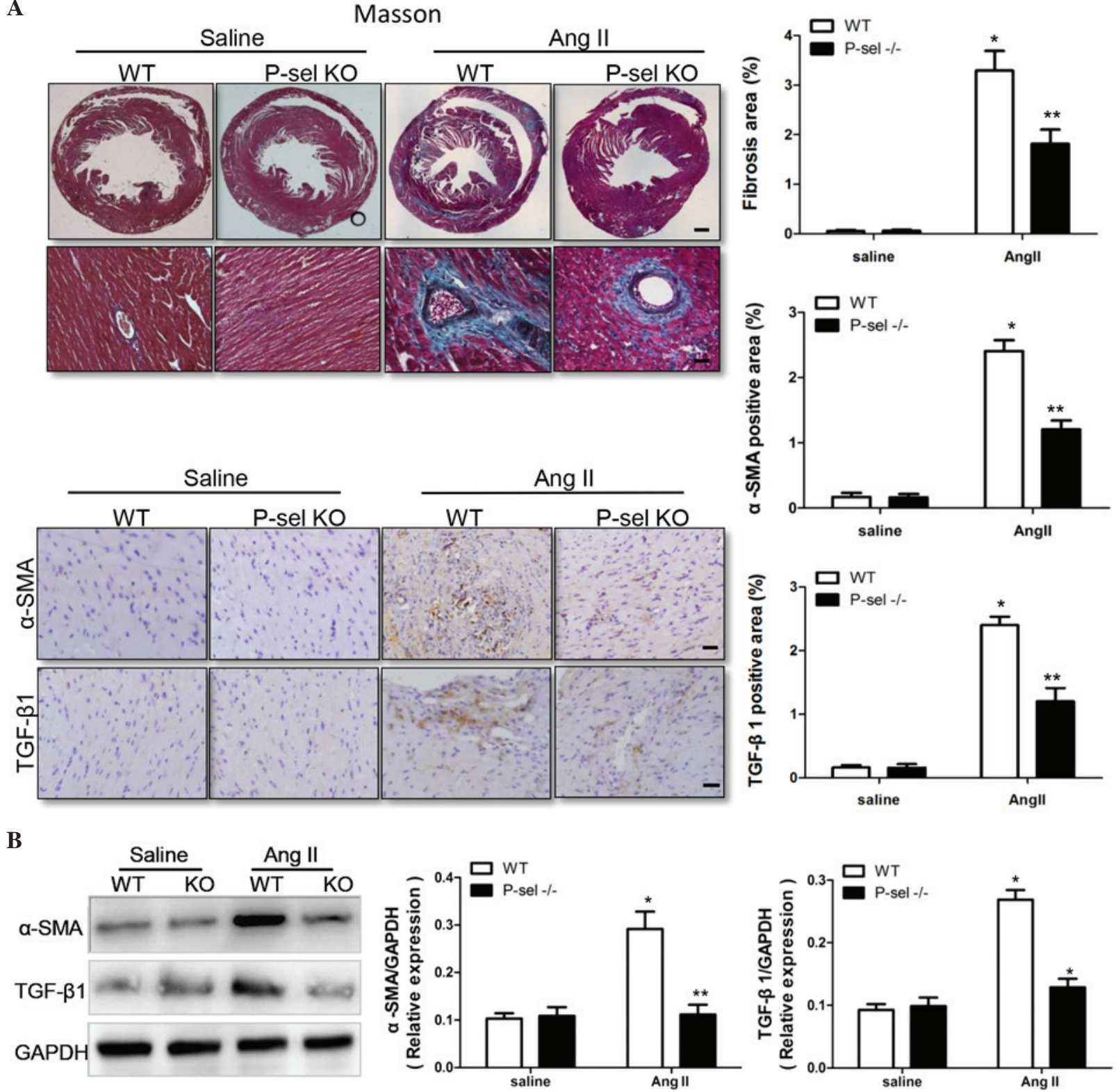

Figure 2. P-selectin deficiency decreases Ang II-induced cardiac fibrosis and inflammation. (A) Cardiac fibrosis in WT and P-sel KO mice following saline or Ang II infusion ( $\mathrm{n}=6 /$ group). At day 7, Masson's trichrome-stained heart sections were analyzed and the fibrotic area was quantified. Scale bars: $500 \mu \mathrm{m}$ (top), $100 \mu \mathrm{m}$ (bottom). Immunohistochemical staining and quantification of $\alpha$-SMA and TGF- $\beta 1$ in the hearts. (B) Western blotting analysis demonstrated the protein expression levels of $\alpha$-SMA and TGF- $\beta 1$. Scale bars: $50 \mu \mathrm{m}$. The data are presented as the mean \pm standard error of the mean (n=6/group). ${ }^{*} \mathrm{P}<0.01$ vs. saline; ${ }^{* *} \mathrm{P}<0.05$ vs. Ang II. $\alpha$-SMA, alpha-smooth muscle actin; TGF- $\beta 1$, transforming growth factor $\beta 1$; WT, wild-type; KO, knockout; Ang II, angiotensin II.

was performed to compare several groups of animals from the transplant experiments. The Mann-Whitney U test was applied to analyze the bleeding time data. SPSS version 17.0 (SPSS, Inc., Chicago, IL, USA) was used to analyze the data. The data are presented as the mean \pm standard error of the mean and $\mathrm{P}<0.05$ was considered to indicate a statistically significant difference.

\section{Results}

Increased P-selectin expression levels were observed in the myocardium and plasma of Ang II-induced hypertensive mice. To investigate the regulation of $\mathrm{P}$-selectin expression in Ang II-induced hypertension, WT mice were infused with Ang II at a dose of $1,500 \mathrm{ng} / \mathrm{kg} / \mathrm{min}$ for 7 days $(12,13)$. P-selectin expression was assessed by western blotting of protein from myocardial samples and by ELISA for plasma samples. P-selectin expression levels were markedly increased in the myocardium and significantly increased in plasma samples following Ang II infusion compared with saline infusion ( $\mathrm{P}<0.01$; Fig. 1A). The SBP was elevated in the WT and P-sel KO groups following Ang II infusion compared with saline infusion $(\mathrm{P}<0.001)$. However, no differences in the SBP were observed between the WT and P-sel KO mice. The left ventricular ejection fraction in the two groups (Fig. 1B) remained unchanged following Ang II infusion. Thus, P-selectin deficiency did not influence SBP or cardiac function after 7 days of Ang II infusion. 
A
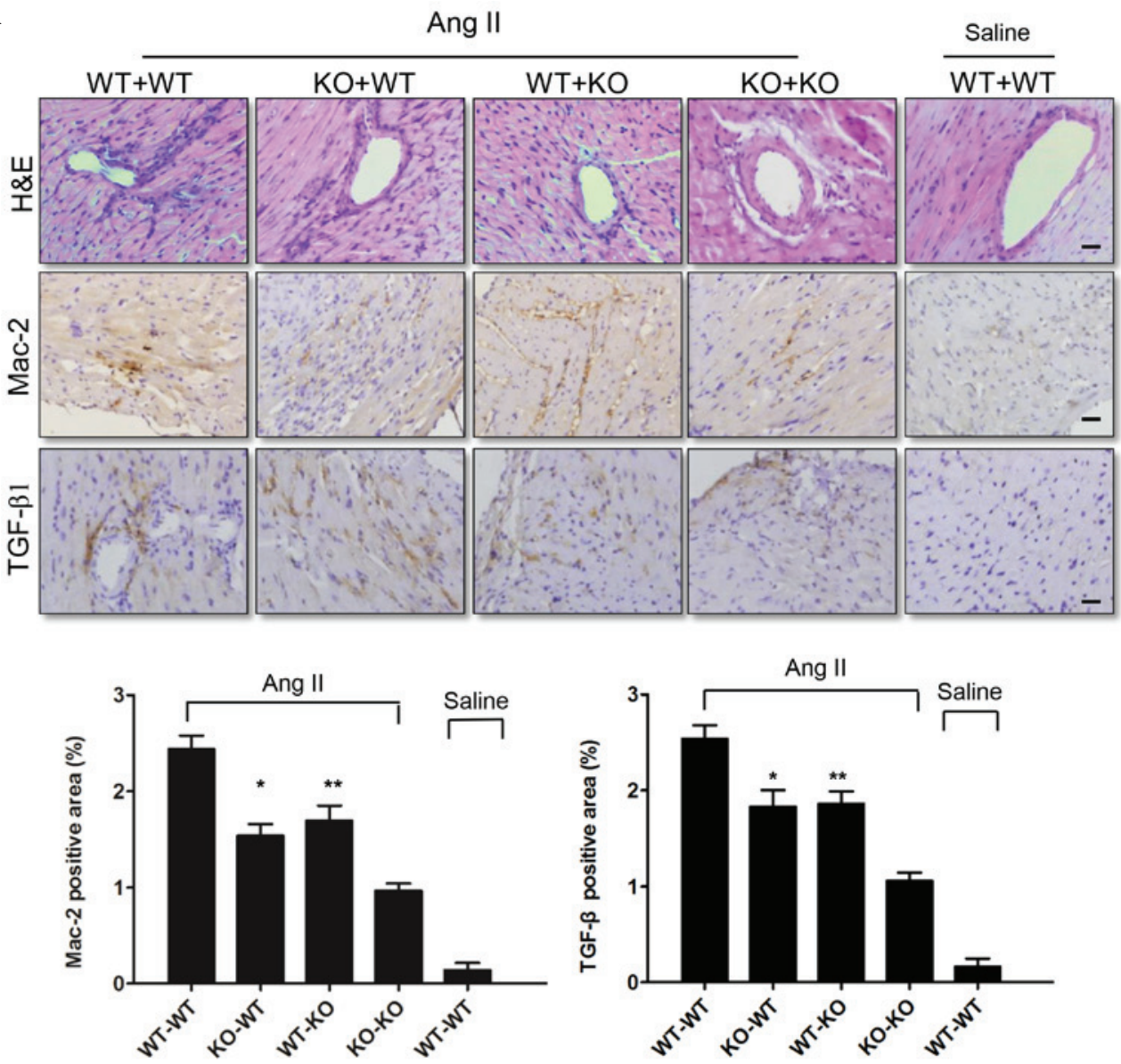

B

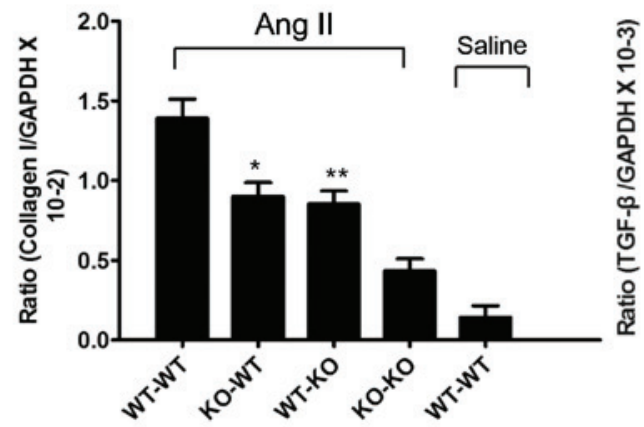

Collagen I mRNA

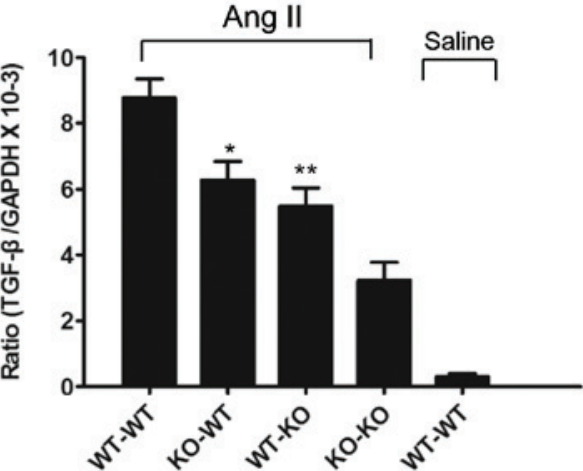

TGF- $\beta 1$ mRNA

Figure 3. P-selectin produced by platelets and endothelial cells affects cardiac fibrosis in Ang II-infused mice. (A) Hematoxylin and eosin staining, immunohistochemical staining, and quantification of Mac-2 and TGF- $\beta 1$ were performed in each group. Among the mice that received a platelet transplantation, the WT-WT group demonstrated the most marked level of fibrosis, and the number of inflammatory cells in the KO-WT and WT-KO groups was similar (B) Reverse transcription-quantitative polymerase chain reaction analysis of collagen I and TGF- $\beta 1$ mRNA expression levels in each group. Scale bars: $50 \mu \mathrm{m}$. The data are presented as the mean \pm standard error of the mean ( $\mathrm{n}=6 /$ group). ${ }^{*} \mathrm{P}<0.05, \mathrm{KO}-\mathrm{WT}$ vs. WT-WT control; ${ }^{* *} \mathrm{P}<0.05, \mathrm{WT}-\mathrm{KO}$ vs. WT-WT control.

P-selectin deficiency decreases cardiac fibrosis. P-sel KO mice were used to examine the effect of P-selectin on Ang II-induced cardiac fibrosis, as assessed using Masson's trichrome staining. Compared with saline infusion, the fibrotic areas were significantly $(\mathrm{P}<0.05)$ larger in the WTP-sel KO hearts following Ang II infusion (Fig. 2A). TGF- $\beta 1$ is an important signal transduction molecule in cardiac fibrosis $(14,15)$, and $\alpha$-SMA increases during the differentiation of fibroblasts into myofibroblasts. TGF- $\beta 1$ and $\alpha$-SMA levels were markedly higher in WT hearts compared with P-sel KO hearts following Ang II infusion, as indicated by immunohistochemistry (Fig. 2A) and by western blotting (Fig. 2B). Thus, P-selectin is critical in cardiac fibrosis in response to Ang II infusion.

Platelet-secreted $P$-selectin is important in the response to Ang II stimulation. A platelet cross-transplantation system was used to determine whether P-selectin predominantly originates from platelets in hypertension-associated cardiac 


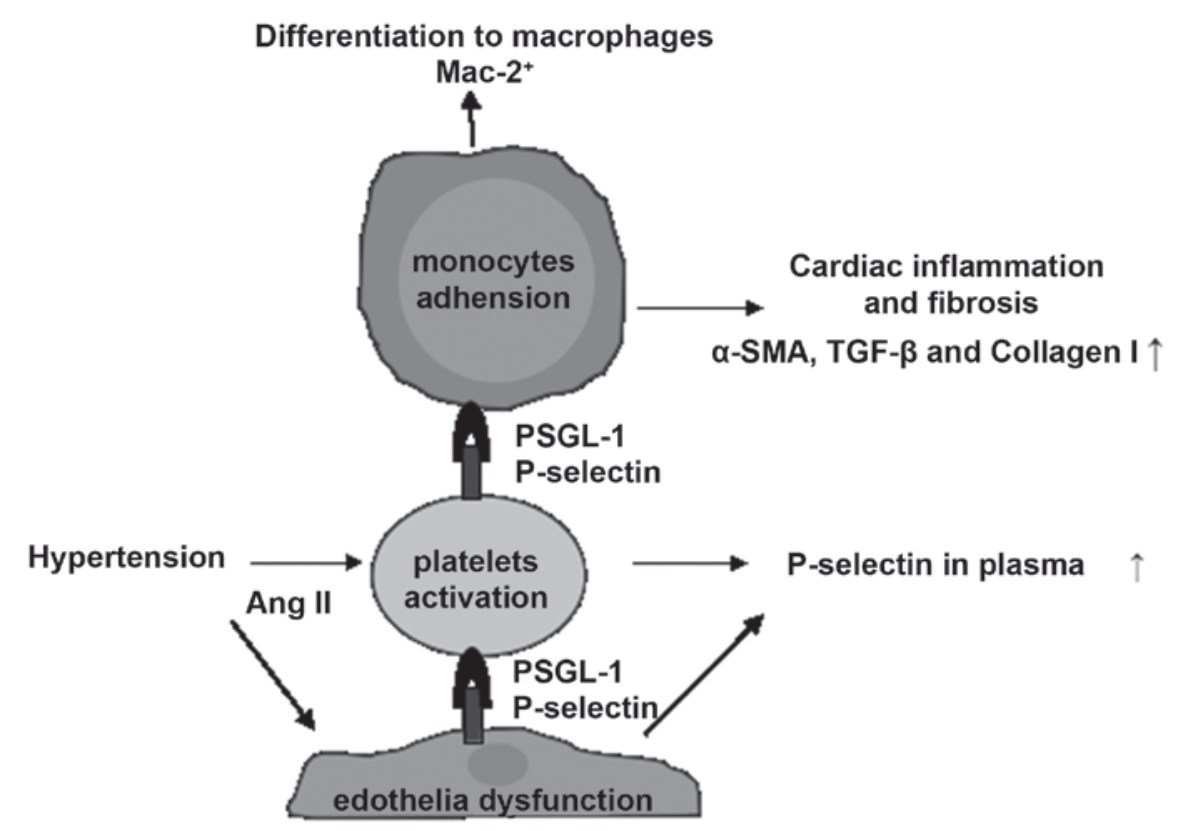

Figure 4. Platelet activation and endothelial dysfunction. Platelets are activated and endothelial function is impaired in Ang II-induced hypertension. P-selectin levels increase in plasma and activated platelets recruit monocytes. Activated platelets interact with PSGL-1 expressed by monocyte via P-selectin. Thus, platelets induce monocyte differentiation into macrophages. Macrophages secrete numerous cytokines and elicit fibroblast differentiation into myofibroblasts, resulting in cardiac inflammation and fibrosis.

inflammation and fibrosis. Platelets $\left[1.5 \mathrm{ml}\left(10^{8} / \mathrm{ml}\right)\right]$ were injected into the tail vein of the recipient mice, which were treated with busulfan for 5 days. A marked difference in cardiac fibrosis was observed between the KO-WT and KO-KO groups, in addition to between the WT-KO and $\mathrm{KO}-\mathrm{KO}$ groups. As presented in Fig. 3A, more inflammatory cells infiltrated the hearts of Ang II-infused KO-WT and WT-KO mice than those of Ang II-infused KO-KO mice. Immunohistochemistry demonstrated that the levels of TGF- $\beta 1$ and the number of Mac-2-positive macrophages in the KO-WT and WT-KO mice were significantly $(\mathrm{P}<0.05)$ lower compared with the WT-WT mice infused with Ang II (Fig. 3A), but significantly greater $(\mathrm{P}<0.05)$ compared with the KO-KO mice. qPCR analysis demonstrated that P-selectin deficiency decreased TGF- $\beta 1$ and collagen I expression (Fig. 3B). Thus, platelet-derived P-selectin increased Ang II-induced cardiac inflammation and fibrosis. However, endothelial cell-derived P-selectin is also involved in hypertension-associated cardiac inflammation and fibrosis.

\section{Discussion}

There is accumulating evidence that $\mathrm{P}$-selectin is a key cytokine in cardiovascular disease (16). A clinical trial demonstrated that P-selectin levels were markedly higher in the plasma of hypertensive patients (17). The results in the current study are consistent with this clinical observation. The plasma levels of P-selectin in WT mice infused with Ang II were significantly higher than those in the control group $(\mathrm{P}<0.01)$. In addition, myocardial P-selectin expression was increased in the Ang II-infused group. Thus, Ang II may stimulate the secretion of P-selectin, which exists in a soluble form in the plasma and heart. Following Ang II infusion, P-selectin expression was induced by the type 1 angiotensin II (AT1) receptor, and this upregulation may be inhibited by the AT1 receptor blocker, valsartan, in hypertensive patients (18). However, the source of soluble P-selectin remains to be elucidated. Plasma soluble P-selectin levels were associated with plasma platelet count and platelet-associated protein expression. However, in a previous study, no association was observed between the expression levels of P-selectin and von Willebrand factor (vWF), which is a marker of activated endothelial cells (19). It was identified that vWF was stored in Weibl-Palade bodies in the endothelial cells, and was secreted into the plasma once the endothelial cells were activated (19). Thus, the majority of the soluble P-selectin was likely secreted by platelets.

Larsson et al (20) observed elevated blood pressure and platelet activation following injection of Ang II into healthy volunteers. Reciprocal activation between platelets and leukocytes promotes the secondary capture of leukocytes (21). Selectin is the predominant leukocyte adhesion molecule. Wang et al (22) demonstrated that impaired leukocyte adhesion in P-sel KO mice was ameliorated by the binding of soluble P-selectin and its ligand, PSGL-1. Myocardial P-selectin expression may be mediated by adhesive leukocytes in the blood. Activated leukocytes express PSGL-1, which binds to P-selectin expressed on endothelial cells or platelet surfaces. These leukocytes then invade cardiac tissue through gaps between endothelial cells to exert pro-inflammatory effects.

Fig. 4 summarizes the signaling pathways involved in the present study. Platelet activation in response to Ang II was recently reported to be an early event that stimulates platelet-leukocyte conjugation and inflammatory cell recruitment into the heart, resulting in cardiac fibrosis (2). However, the mechanism by which platelets mediate inflammation remained to be elucidated. Results from the present study demonstrate direct evidence that P-selectin, a marker of platelet activation, is involved in Ang II-induced 
hypertension-associated cardiac inflammation and fibrosis. Western blotting and immunohistochemistry demonstrated that the expression levels of the profibrotic cytokine, TGF- $\beta 1$, and the fibroblast differentiation marker, $\alpha$-SMA, was decreased in the P-sel KO mice, suggesting that P-selectin is key in cardiac fibrosis following Ang II infusion. Consistently, the number of Mac-2-positive inflammatory cells, which are upregulated under inflammatory conditions, were markedly reduced in the $\mathrm{P}$-sel $\mathrm{KO}$ mice (KO-KO vs. WT-WT), indicating that P-selectin deficiency decreased Ang II-induced cardiac inflammation. However, cardiac function was not affected after 7 days of Ang II infusion in the P-sel KO mice, which was consistent with previous studies demonstrating that cathepsin S, intercellular adhesion molecule 1 and IL-6 deficiencies did not alter the Ang II-induced elevated blood pressure and cardiac dysfunction $(11,23)$. A 4-week-long Ang II infusion period induced cardiac hypertrophy, remodeling and dysfunction in mice $(24,25)$, whereas short-term Ang II infusion (7 days) may induce cardiac inflammation but is insufficient to result in cardiac dysfunction. The focus of the current study was to investigate the role of platelet P-selectin in the early stage of hypertensive cardiac inflammation and fibrosis, thus, a short-term Ang II infusion animal model was selected.

Clinical and animal studies have reported platelet activation and inflammation in hypertensive mice (26) and patients (27-29). Injection of Ang II may induce platelet activation in humans and mice $(16,26)$. The binding of P-selectin to PSGL-1 mediates platelet-leukocyte conjugation in early-stage myocardial infarction, resulting in cardiac inflammation and remodeling (30). In a previous study on the murine cutaneous Arthus reaction, platelets regulated leukocyte recruitment in a P-selectin/PSGL-1 interaction-dependent manner (31). Numerous studies have illustrated that circulating platelet activation and platelet-leukocyte conjugation are key inflammatory mediators. P-selectin-deficient conditions may be rescued with WT platelets. In the present study, using platelet cross-transplantation demonstrated that the P-selectin involved in Ang II-induced hypertension-associated cardiac inflammation and fibrosis predominantly originated from platelets. The KO-WT group (injection of activated WT platelets) exhibited increased inflammatory cells and fibrotic tissue compared with the KO-KO group (injection of $\mathrm{P}$-sel $\mathrm{KO}$ platelets) but the fibrotic tissue was reduced compared with the WT-WT group.

Monocyte recruitment is regulated by activated platelets and platelet-derived P-selectin in response to Ang II-induced hypertension. Monocytes are recruited into the cardiac tissue, where they transform into macrophages, which secrete numerous cytokines and elicit fibroblast differentiation into myofibroblasts within the myocardial infarction area (32-34). The activated myofibroblasts release an abundance of extracellular matrix proteins, including collagen I/III and fibronectin, thus inducing cardiac fibrosis (35-37).

The WT-KO group, which predominantly demonstrated the effects of endothelial cell-derived P-selectin, exhibited a similar trend to the KO-WT group. This suggests that in addition to platelets, endothelial cells and endothelial cell-derived P-selectin affects the hypertension-associated cardiac fibrosis. The Ang II-induced elevated blood pressure resulted in platelet activation and endothelial dysfunction, leading to P-selectin secretion into the plasma. Similarly, in atherosclerosis, platelet- and endothelial cell-derived P-selectin promote the progression of atherosclerotic lesion development (8).

In conclusion, P-selectin expression levels were increased in myocardium and plasma samples from Ang II-infused mice, and P-selectin deficiency reduced cardiac inflammation and fibrosis. P-selectin produced by activated platelets and by endothelial cells during Ang II stimulation resulted in the development of hypertension-associated cardiac inflammation and fibrosis.

\section{Acknowledgements}

The present study was supported by grants from the National Natural Science Foundation of China (grant no. 81070090).

\section{References}

1. Marvar PJ, Thabet SR, Guzik TJ, Lob HE, McCann LA, Weyand C, Gordon FJ and Harrison DG: Central and peripheral mechanisms of T-lymphocyte activation and vascular inflammation produced by angiotensin II-induced hypertension. Circ Res 107: 263-270, 2010.

2. Jia LX, Qi GM, Liu O, Li TT, Yang M, Cui W, Zhang WM, Qi YF and Du J: Inhibition of platelet activation by clopidogrel prevents hypertension-induced cardiac inflammation and fibrosis. Cardiovasc Drugs Ther 27: 521-530, 2013.

3. Mantovani A and Garlanda C: Platelet-macrophage partnership in innate immunity and inflammation. Nat Immunol 14: 768-770, 2013.

4. van Gils JM, Zwaginga JJ and Hordijk PL: Molecular and functional interactions among monocytes, platelets, and endothelial cells and their relevance for cardiovascular diseases. J Leukoc Biol 85: 195-204, 2009.

5. von Hundelshausen $P$ and Weber C: Platelets as immune cells: Bridging inflammation and cardiovascular disease. Circ Res 100: 27-40, 2007.

6. National Research Council (US) Institute for Laboratory Animal Research: Guide for the Care and Use of Laboratory Animals. National Academies Press, Washington, DC, pp85-23, 1996.

7. Gresele P, Momi S, Berrettini M, Nenci GG, Schwarz HP Semeraro $\mathrm{N}$ and Colucci $\mathrm{M}$ : Activated human protein $\mathrm{C}$ prevents thrombin-induced thromboembolism in mice. Evidence that activated protein $\mathrm{c}$ reduces intravascular fibrin accumulation through the inhibition of additional thrombin generation. J Clin Invest 101: 667-676, 1998.

8. Burger PC and Wagner DD: Platelet P-selectin facilitates atherosclerotic lesion development. Blood 101: 2661-2666, 2003.

9. Huang XR, Chung AC, Yang F, Yue W, Deng C, Lau CP, Tse HF and Lan HY: Smad3 mediates cardiac inflammation and fibrosis in angiotensin II-induced hypertensive cardiac remodeling. Hypertension 55: 1165-1171, 2010.

10. Livak KJ and Schmittgen TD: Analysis of relative gene expression data using real-time quantitative PCR and the 2(-Delta Delta C(T)) method. Methods 25: 402-408, 2001.

11. Ma F, Li Y, Jia L, Han Y, Cheng J, Li H, Qi Y and Du J: Macrophage-stimulated cardiac fibroblast production of IL-6 is essential for TGF $\beta /$ Smad activation and cardiac fibrosis induced by angiotensin II. PLoS One 7: e35144, 2012.

12. Usher MG, Duan SZ, Ivaschenko CY, Frieler RA, Berger S, Schütz G, Lumeng CN and Mortensen RM: Myeloid mineralocorticoid receptor controls macrophage polarization and cardiovascular hypertrophy and remodeling in mice. J Clin Invest 120: 3350-3364, 2010.

13. Totani L and Evangelista V: Platelet-leukocyte interactions in cardiovascular disease and beyond. Arterioscler Thromb Vasc Biol 30: 2357-2361, 2010.

14. Honsho S, Nishikawa S, Amano K, Zen K, Adachi Y, Kishita E, Matsui A, Katsume A, Yamaguchi S, Nishikawa K, et al: Pressure-mediated hypertrophy and mechanical stretch induces IL-1 release and subsequent IGF-1 generation to maintain compensative hypertrophy by affecting Akt and JNK pathways. Circ Res 105: 1149-1158, 2009. 
15. Bujak M, Dobaczewski M, Chatila K, Mendoza LH, Li N Reddy A and Frangogiannis NG: Interleukin-1 receptor type I signaling critically regulates infarct healing and cardiac remodeling. Am J Pathol 173: 57-67, 2008.

16. Guo L, Sun G, Wang G, Ning W and Zhao K: Soluble P-selectin promotes acute myocardial infarction onset but not severity. Mol Med Rep 11: 2027-2033, 2015.

17. Spencer CG, Gurney D, Blann AD, Beevers DG and Lip GY; ASCOT Steering Committee, Anglo-Scandinavian Cardiac Outcomes Trial: Von willebrand factor, soluble P-selectin, and target organ damage in hypertension: A substudy of the anglo-scandinavian cardiac outcomes trial (ASCOT). Hypertension 40: 61-66, 2002.

18. Piqueras L, Kubes P, Alvarez A, O'Connor E, Issekutz AC, Esplugues JV and Sanz MJ: Angiotensin II induces leukocyte-endothelial cell interactions in vivo via AT(1) and AT(2) receptor-mediated P-selectin upregulation. Circulation 102: 2118-2123, 2000.

19. Kawai Y and Montgomery RR: Endothelial cell processing of von Willebrand proteins. Ann N Y Acad Sci 509: 60-70, 1987.

20. Larsson PT, Schwieler JH and Wallén NH: Platelet activation during angiotensin II infusion in healthy volunteers. Blood Coagul Fibrinolysis 11: 61-69, 2000.

21. Kuebler WM: Selectins revisited: The emerging role of platelets in inflammatory lung disease. J Clin Invest 116: 3106-3108, 2006.

22. Wang HB, Wang JT, Zhang L, Geng ZH, Xu WL, Xu T, Huo Y, Zhu X, Plow EF, Chen M and Geng JG: P-selectin primes leukocyte integrin activation during inflammation. Nat Immunol 8: 882-892, 2007.

23. Pan L, Li Y, Jia L, Qin Y, Qi G, Cheng J, Qi Y, Li H and Du J: Cathepsin $\mathrm{S}$ deficiency results in abnormal accumulation of autophagosomes in macrophages and enhances Ang II-induced cardiac inflammation. PLoS One 7: e35315, 2012.

24. Dai DF, Johnson SC, Villarin JJ, Chin MT, Nieves-Cintrón M, Chen T, Marcinek DJ, Dorn GW II, Kang YJ, Prolla TA, et al Mitochondrial oxidative stress mediates angiotensin II-induced cardiac hypertrophy and Galphaq overexpression-induced heart failure. Circ Res 108: 837-846, 2011.

25. Izumiya Y, Kim S, Izumi Y, Yoshida K, Yoshiyama M, Matsuzawa A, Ichijo $\mathrm{H}$ and Iwao $\mathrm{H}$ : Apoptosis signal-regulating kinase 1 plays a pivotal role in angiotensin II-induced cardiac hypertrophy and remodeling. Circ Res 93: 874-883, 2003.

26. Bujak M, Dobaczewski M, Chatila K, Mendoza LH, Li N, Reddy A and Frangogiannis NG: Interleukin-1 receptor type I signaling critically regulates infarct healing and cardiac remodeling. Am J Pathol 173: 57-67, 2008.
27. Ferroni P, Guagnano MT, Falco A, Paoletti V, Manigrasso MR, Michetti N, Santilli F, Guadagni F, Basili S and Davì G: Association of low-grade inflammation and platelet activation in patients with hypertension with microalbuminuria. Clin Sci (Lond) 114: 449-455, 2008.

28. Davì G, Gresele P, Violi F, Basili S, Catalano M, Giammarresi C, Volpato R, Nenci GG, Ciabattoni G and Patrono C: Diabetes mellitus, hypercholesterolemia, and hypertension but not vascular disease per se are associated with persistent platelet activation in vivo. Evidence derived from the study of peripheral arterial disease. Circulation 96: 69-75, 1997.

29. Nadar SK, Blann AD, Kamath S, Beevers DG and Lip GY: Platelet indexes in relation to target organ damage in high-risk hypertensive patients: A substudy of the anglo-scandinavian cardiac outcomes trial (ASCOT). J Am Coll Cardiol 44: 415-422, 2004

30. Liu Y, Gao XM, Fang L, Jennings NL, Su Y, Q X, Samson AL, Kiriazis H, Wang XF, Shan L, et al: Novel role of platelets in mediating inflammatory responses and ventricular rupture or remodeling following myocardial infarction. Arterioscler Thromb Vasc Biol 31: 834-841, 2011.

31. Hara T, Shimizu K, Ogawa F, Yanaba K, Iwata Y, Muroi E, Takenaka M, Komura K, Hasegawa M, Fujimoto M and Sato S: Platelets control leukocyte recruitment in a murine model of cutaneous arthus reaction. Am J Pathol 176: 259-269, 2010.

32. Larsen E, Celi A, Gilbert GE, Furie BC, Erban JK, Bonfanti R, Wagner DD and Furie B: PADGEM protein: A receptor that mediates the interaction of activated platelets with neutrophils and monocytes. Cell 59: 305-312, 1989.

33. Theilmeier G, Lenaerts T, Remacle C, Collen D, Vermylen J and Hoylaerts MF: Circulating activated platelets assist THP-1 monocytoid/endothelial cell interaction under shear stress. Blood 94: 2725-2734, 1999.

34. Mollmann H, Nef HM and Troidl C: Turning the right screw: Targeting the interleukin- 6 receptor to reduce unfavourable tissue remodelling after myocardial infarction. Cardiovasc Res 87: 395-396, 2010

35. Staufenberger S, Jacobs M, Brandstatter K, Hafner M, Regitz-Zagrosek V, Ertl G and Schorb W: Angiotensin II type 1 receptor regulation and differential trophic effects on rat cardiac myofibroblasts after acute myocardial infarction. J Cell Physiol 187: 326-335, 2001.

36. Lijnen PJ, Petrov VV and Fagard RH: Angiotensin II-induced stimulation of collagen secretion and production in cardiac fibroblasts is mediated via angiotensin II subtype 1 receptors. J Renin Angiotensin Aldosterone Syst 2: 117-122, 2001

37. Hafizi S, Wharton J, Morgan K, Allen SP, Chester AH, Catravas JD, Polak JM and Yacoub MH: Expression of functional angiotensin-converting enzyme and AT1 receptors in cultured human cardiac fibroblasts. Circulation 98: 2553-2559, 1998. 